\title{
Placental drug transport-on-a-chip: a microengineered in vitro model of transporter-mediated drug efflux in the human placental barrier
}

\author{
Cassidy Blundell ${ }^{1}$, Yoon-Suk Yi ${ }^{1}$, Lin Ma ${ }^{1}$, Emily R. Tess ${ }^{1}$, Megan J. Farrell ${ }^{1}$, Andrei \\ Georgescu $^{1}$, Lauren M. Aleksunes ${ }^{2}$, and Dongeun Huh ${ }^{1, *}$ \\ ${ }^{1}$ Department of Bioengineering, School of Engineering and Applied Science, University of \\ Pennsylvania, PA \\ ${ }^{2}$ Department of Pharmacology and Toxicology, Rutgers University Ernest Mario School of \\ Pharmacy, Piscataway, NJ
}

\begin{abstract}
The current lack of knowledge about the effect of maternally administered drugs on the developing fetus is a major public health concern worldwide. The first critical step towards predicting the safety of medications in pregnancy is to screen drug compounds for their ability to cross the placenta. However, this type of preclinical study has been hampered by the limited capacity of existing in vitro and ex vivo models to mimic physiological drug transport across the maternalfetal interface in the human placenta. Here we demonstrate the proof-of-principle for utilizing a microengineered model of the human placental barrier to simulate and investigate drug transfer from the maternal to the fetal circulation. Using the gestational diabetes drug glyburide as a model compound, we show that our microphysiological system is capable of reconstituting efflux transporter-mediated active transport function of the human placental barrier to limit fetal exposure to maternally administered drugs. Our data provide evidence that the placenta-on-a-chip may serve as a new screening platform to enable more accurate prediction of drug transport in the human placenta.
\end{abstract}

\section{Introduction}

Fetal exposure to medications is a critical issue in pregnancy that can lead to serious gestational complications and long-term health consequences. A recent survey of clinical data from 1976 to 2008 reported that approximately 90\% of women in the United States take at least one medication during pregnancy ${ }^{[1]}$. Moreover, retrospective studies have reported significantly increasing use of over-the-counter and prescription drugs by pregnant women. In the United States, for instance, the fraction of women taking four or more medications during pregnancy has more than doubled over the past three decades and continues to rise $^{[2]}$. Despite this alarming trend, the risks posed by maternally administered drugs to the developing fetus remain a largely unanswered question to both clinicians and expecting mothers.

\footnotetext{
*To whom correspondence should be addressed: huhd@ seas.upenn.edu, Tel: 1-215-898-5208.
} 
One of the major contributors to this problem is our limited ability to model and predict the physiological transport function of the human placenta. The placenta is responsible for regulating the transfer of all materials between the mother and the fetus during pregnancy, and therefore plays an essential role in fetal exposure to drugs ${ }^{[3]}$. To assess the safety of medications in pregnancy appropriately, it is imperative to first understand whether and how drug molecules cross the placental barrier from the maternal to the fetal circulation. While animal models of pregnancy provide useful surrogates for these types of studies, considerable interspecies differences in placental structure and function have rendered the use of these models controversial ${ }^{[4,5]}$. The inadequacy of preclinical animal testing is best highlighted by the tragedy of thalidomide in the 1960s that caused serious birth defects and fetal deaths due to the failure of animal models to predict human fetal toxicity ${ }^{[6]}$. Despite significant progress in animal experimentation for reproductive toxicology, the predictive value of animal data continues to be questionable. As a result, alternative strategies are urgently needed for the preclinical assessment and accurate prediction of drug transfer across the human placenta and subsequent fetal exposure.

To address this critical unmet need, here we demonstrate the proof-of-principle for using a human cell-based microphysiological model of the placenta for simulation and quantitative analysis of placental drug transport. This study builds upon the "placenta-on-a-chip" system that we have developed previously to mimic the salient structure and function of the human placental barrier (Figure 1A-C ${ }^{[7]}$. In this microdevice, human trophoblast cells and villous endothelial cells are cultured in apposition on a semipermeable membrane under flow conditions (Figure 1D-E). This configuration makes it possible to reconstitute the multilayered architecture and hemodynamic environment of the placental barrier, providing compelling advantages over existing in vitro models based on static culture of trophoblast monolayers in Transwell inserts ${ }^{[8]}$. In comparison to traditional ex vivo approaches using extracorporeal perfusion of cannulated whole human placenta ${ }^{[9,10]}$, our model is more attractive for mechanistic investigation due to its ability to precisely control and manipulate various key parameters of placental drug transport.

In the present study, we leverage these unique capabilities to recapitulate physiological function of the human placental barrier to regulate maternal-to-fetal transfer of drugs that are commonly used during pregnancy. A specific focus of our investigation is on active drug transport mediated by the breast cancer resistance protein (BCRP), which is one of the major ATP-binding cassette (ABC) efflux transporters abundantly expressed in the apical membrane of trophoblast cells in the placenta ${ }^{[11]}$. Studies have shown that BCRP plays a central role in limiting fetal drug exposure by pumping various types of maternally administered drug compounds from the fetal compartment back into the maternal circulation ${ }^{[12]}$. We demonstrate the ability of our placenta-on-a-chip model to mimic this critical BCRP-mediated transport function using a model drug, glyburide - a BCRP substrate and an oral medication used to treat diabetes in pregnancy. Our data show that the in vitro human placental barrier engineered in our device reconstitutes physiological expression of BCRP in trophoblast cells and emulates the protective role of its in vivo counterpart by effectively preventing transfer of glyburide from the maternal to the fetal compartment. 
The work described herein represents an important step towards the development of humanrelevant predictive in vitro models for preclinical evaluation of drug safety in pregnancy. The placenta-on-a-chip system holds great potential as a promising alternative to traditional models in reproductive toxicology and offers a novel platform to screen drug transport in the human placenta in a rapid and cost-effective manner. We believe that our technology provides a technical basis for more systematic investigations in the future and may eventually allow clinicians to make informed decisions and recommendations to pregnant mothers.

\section{Results and Discussion}

\subsection{Production of the microengineered human placental barrier}

As illustrated in Figure 1C, the in vivo placental barrier is a multilayered structure that consists of trophoblast and fetal endothelial cells, which are separated by basement membrane. As pregnancy progresses, this villous membrane becomes gradually thinner and reaches an average thickness of approximately $4.53 \mu \mathrm{m}$ at term, as determined by measurements made in placentas following cesarean delivery ${ }^{[13]}$. Formation of appropriate intercellular junctions in these tissue layers is crucial for physiological barrier function of the placenta to mediate transfer of substances in the maternal blood from the intervillous space to the fetal capillaries. Perfusion co-culture of human trophoblasts and human placental villous endothelial cells (HPVECs) in our compartmentalized microfluidic device provided a means to recapitulate this important feature of the placental barrier. The cells plated on either side of a fibronectin-coated semipermeable membrane were observed to proliferate continuously and form confluent monolayers within 24 hours of cell seeding. By 72 hours of culture, the HPVECs had grown out of the membrane surface to cover all four channel walls in the lower microchannel. Typically, the cells were cultured for 3 days under continuous perfusion of medium to establish a bi-layer tissue structure reminiscent of the trophoblast-endothelial interface of the placental barrier in vivo. Importantly, immunofluorescence imaging illustrated the formation of continuous intercellular junctional complexes in both cell types. The trophoblast cell and HPVEC populations showed robust expression of E-cadherin and vascular endothelial (VE)-cadherin, respectively (Figure 2A,B). Our analysis revealed no visible intercellular gaps, suggesting the formation of continuous monolayers on either side of the membrane. The structural integrity of the microengineered barrier was confirmed by our analysis of electrical resistance across the trophoblast-endothelial interface. This study showed that the transepithelial-endothelial electrical resistance (TEER) measured in the co-culture system increased rapidly over time and reached approximately $5020 \Omega \cdot \mathrm{cm}^{2}$ when the cells were allowed sufficient time (typically over 2 days) to form fully confluent monolayers.

To further assess barrier function of our model, we also conducted a permeability assay to measure the passage of FITC-conjugated inulin across the microengineered placental barrier. Control experiments using acellular devices revealed significant transfer of FITC-inulin from the maternal to the fetal compartments, as evidenced by more than $25 \%$ increase in the fluorescence intensity of fetal outflow over a period of 3 hours (Figure 2C). In contrast, the co-culture model showed no detectable florescence in the fetal chamber, illustrating 
negligible transport of FITC-inulin across the trophoblast-endothelial barrier. Combined with the intercellular junction staining data and TEER measurements, these results clearly demonstrate the formation of a microengineered tissue-tissue interface with structural integrity and appropriate barrier function.

Another key feature of the human placental barrier that influences drug transfer is the microvilli on the apical surface of the syncytiotrophoblast. These cellular protrusions serve to increase the overall placental surface area for maternal-to-fetal transport, which increases significantly over the course of gestation ${ }^{[14]}$. Our microengineered model enabled reproduction of this physiological phenotype as well. In microscopic visualization using actin staining, we observed that fine hair-like projections covered the entire cell surface of the vast majority of trophoblast cells cultured in our microdevice (Figure 2D). Although intercellular variability was observed in their size and density, these microvilli were generated on the apical side of the cells facing the maternal compartment, matching the polarity of syncytiotrophoblast microvilli in vivo (Figure 2E). Previously we have shown that the extent of microvilli formation is reduced considerably when trophoblasts are cultured on a similar semipermeable membrane support in a static Transwell insert ${ }^{[7]}$. In conjunction with this previous finding, our results indicate that dynamic culture conditions due to continuous perfusion in our model is beneficial not only for maintaining cell viability but also for inducing cultured trophoblasts to recapitulate their native morphological characteristics. Considering that a variety of active membrane transporters implicated in the transfer of drugs and xenobiotics are expressed on the microvillous surface of trophoblast cells $^{[15]}$, the formation of abundant microvilli demonstrated here represents a critical requirement for reconstituting physiological drug transport across the placental barrier.

\subsection{Induction and characterization of trophoblast syncytialization}

The placental barrier undergoes continuous and significant alterations in its structure during pregnancy ${ }^{[16]}$. This structural evolution allows for more efficient exchange of vital substances between the mother and the fetus while enhancing barrier function of the placenta to further restrict the penetration of foreign materials into the fetal circulation ${ }^{[17]}$. Central to this physiological development is progressive intercellular fusion of cytotrophoblasts on the maternal surface of the chorionic villi that eventually leads to the formation of a multinucleated barrier termed the syncytiotrophoblast ${ }^{[18]}$. Given the integral role of trophoblast cells in placental drug transport, the process of syncytialization is an important feature that needs to be recapitulated in in vitro platforms for screening the safety of drugs for use in pregnancy.

To induce trophoblast syncytialization in our model, we used forskolin to increase intracellular levels of cyclic adenosine monophosphate (cAMP) in the BeWo b30 clone ${ }^{[19]}$. When these cells were perfused with forskolin-containing medium, progressive cell fusion occurred over the duration of treatment (Figure 2F). In the first 24 hours, most of the cells retained a cytotrophoblast-like morphology but prolonged treatment resulted in noticeable changes characterized by cytoplasmic fusion and nuclear aggregation in a significant fraction of the cells. Our quantitative morphometric analysis showed more than a $38 \%$ increase in average cell area from 24 to 72 hours, indicating forskolin-induced cell fusion 
(Figure 2G). Aggregation of cell nuclei was also evident from a significant increase in the nuclear area that occurred during the same period $(\mathrm{p}<0.0001)$ (Figure $2 \mathrm{H})$. Although syncytialization of the entire monolayer was not achievable even in further extended treatment, this method serves to significantly improve the physiological relevance of our model and also provides the opportunity to more realistically simulate drug transfer across the native placental barrier.

\subsection{Heparin transport across the placental barrier}

Prior to investigating active drug transport using the placenta-on-a-chip model, we first examined whether our microengineered placental tissue had the capacity to form an effective barrier to soluble molecules and to properly mimic restrictive barrier function of the maternal-fetal interface in vivo. Specifically, we used fluorescently labeled heparin as a tracer to analyze molecular transport from the maternal compartment to the fetal microchannel. Heparin is an anticoagulant medication used in the treatment of deep vein thrombosis and pulmonary embolism during pregnancy ${ }^{[20]}$. It has been established that heparin does not cross the placental barrier due to its large molecular size (3000-15000 $\mathrm{kDa}$ ), offering a safe alternative to other anticoagulants, such as warfarin, which can be transferred to the fetal circulation and exert deleterious effects on the developing fetus ${ }^{[21,22]}$.

In our study, we exploited this non-permeable property of heparin to assess barrier integrity of the microengineered placental interface. Our analysis was carried out by administering fluorescently conjugated heparin into the maternal compartment and subsequently measuring the fluorescence intensity of outflow collected from both microchannels to quantify heparin transport. As a control condition, we first tested acellular devices that consisted of bare semipermeable membrane supports without trophoblast cells and HPVECs. In this case, the fetal perfusate sampled over the course of 5 hours showed high levels of fluorescence, corresponding to $11.3 \%$ of the initial heparin concentration in the maternal compartment (Figure 3A). When the same assay was conducted in the co-culture system, however, fluorescence intensity of collected samples was reduced dramatically and was negligible as compared to that measured in the acellular model ( $\mathrm{p}<0.01$ ) (Figure 3A). Under this condition, the final fetal concentration of fluorescein-heparin was estimated to be approximately $0.2 \%$ of the initial maternal concentration. These data indicate that our microengineered tissue can effectively prevent the passage of heparin into the fetal compartment, confirming previous findings about heparin transport in the human placenta. Our results also suggest the ability of our model to recapitulate the barrier integrity that prevents the passage of heparin across the placental barrier in vivo.

\subsection{Active drug transport mediated by the microengineered placental barrier}

In the next phase of the study, we explored the possibility of simulating active drug transport function of the placental barrier in our microfluidic device. For this investigation, we used glyburide as a representative example of a medication commonly prescribed and administered to pregnant women. Glyburide is a second-generation sulfonylurea widely used in the treatment of gestational diabetes, which is a condition that affects up to $10 \%$ of pregnancies in the United States and is becoming increasingly prevalent worldwide ${ }^{[23-25]}$. With the rapidly growing use of diabetes drugs in pregnancy, extensive research has been 
undertaken in the past decades to evaluate the transfer of glyburide in the human placenta. Several ex vivo perfusion and clinical studies have reported that maternally administered glyburide is nearly undetectable or found in very low concentrations in the fetal circulation even at concentrations far exceeding therapeutic doses ${ }^{[26,27]}$. Increasing evidence now suggests that the limited placental transfer of glyburide is achieved through the function of $\mathrm{ABC}$ efflux transporters expressed by trophoblasts that actively pump the drug against a concentration gradient into the maternal circulation ${ }^{[28-30]}$. Based on this knowledge, we asked whether our placenta-on-a-chip model was capable of mimicking active transport of glyburide mediated by BCRP, which is one of the most important and abundant efflux transporters in the human placenta ${ }^{[31]}$.

First, we measured and characterized the expression of BCRP in the microengineered placental barrier. When syncytialized trophoblast cells were immunostained and imaged using confocal microscopy, they showed robust BCRP expression throughout the monolayer (Figure 3B). Importantly, transporter expression was localized to the apical microvillous surface of the cells (Figure 3C), closely matching the spatial distribution of BCRP reported in vivo $^{[11]}$.

To investigate glyburide transport, we then introduced culture medium containing $1 \mu \mathrm{M}$ BODIPY-conjugated glyburide into the upper maternal channel and monitored the fluorescence of perfusate retrieved from the fetal compartment (Figure 3D). Both sides of the barrier were perfused continuously throughout the duration of the experiments to simulate the hemodynamic environment of the placenta in vivo. In the absence of cells cultured on the membrane support, the concentration of glyburide in the maternal perfusate remained virtually constant without significant fluctuations over time (Figure 3E). In comparison to this acellular model, the BeWo-HPVEC barrier showed an entirely different temporal profile of the maternal drug concentration. The concentration of glyburide at early time points were measured to be considerably lower (e.g., 5-fold reduction at $30 \mathrm{~min}$ ) (Figure 3E), indicating a significant loss of drug from the maternal circulation. We suspect that this is presumably due to drug uptake by the trophoblast cells facing the maternal side, as evidenced by strong green fluorescence detected in this cell population after 3-hour perfusion with BODIPY-labeled glyburide (Figure 3F). It should be noted that PDMS can absorb glyburide (Supplementary Figure 1) and thus may serve as another source of drug loss from the maternal flow ${ }^{[32]}$. Considering that the acellular device made out of the same material still yielded high maternal glyburide concentrations, however, the contribution of PDMS drug absorption may not be as significant as cellular uptake.

Interestingly, the initially low concentration of glyburide began to rise and continued to increase over time, eventually reaching the levels that were almost 4 times higher by the end of 3-hour drug perfusion (Figure 3F). We speculate that this significant increase is attributed to BCRP-mediated active transport of BODIPY-glyburide absorbed by the placental barrier back into the maternal circulation. Another important observation was that the fetal concentration of glyburide remained constant and considerably lower than the maternal concentration throughout perfusion. Additionally, microscopic imaging of HPVECs after drug treatment yielded no detectable intracellular fluorescence (Figure 3G). These results were compared to glyburide transport in endothelial monoculture conditions, where only 
HPVECs were present on the lower surface of the polycarbonate membrane. Under these conditions, the progressive increase in maternal drug concentration seen in the co-cultured placental barrier was no longer observed (Figure $3 \mathrm{H}$ ). The level of glyburide in the maternal compartment was maintained low without significant changes over the entire duration of drug perfusion. Considering that BCRP is absent in the endothelial cell population ${ }^{[11]}$, this observation indicates that the transporter-mediated efflux of maternally administered glyburide is governed predominantly by BeWo cells in the microengineered placental barrier. Taken together, our results clearly illustrate the insignificant transfer of glyburide across the microengineered placental barrier.

\subsection{Biochemical inhibition of glyburide transport}

To validate the active transport function of BCRP in our glyburide transport model, we next measured the maternal concentration of BODIPY glyburide in the presence of Ko143, which is a specific inhibitor of $\mathrm{BCRP}^{[33,34]}$. In these experiments, addition of $150 \mathrm{nM}$ Ko143 to the glyburide-containing medium resulted in the reduction of fluorescence detected in the maternal perfusate, as compared to the inhibitor-free condition (Figure 4A). This change was statistically significant from 90 to 150 minutes of perfusion. Considering that no structural changes of the placental barrier were observed during this period and identical hydrodynamic conditions were maintained across all experiments, these data suggest that Ko143 effectively reduced the transport function of BCRP and decreased the amount of glyburide shuttled back into the maternal compartment from the BeWo cells. This observation was further supported by increased intracellular green fluorescence in the BeWo cells treated with Ko143 (Figure 4B). Despite this significant effect of a BCRP inhibitor, however, no visible fluorescence was detected in the HPVEC population cultured on the other side of the membrane, nor did the fetal concentration of glyburide significantly increase over time (Figure 4C). This result implies that the reduced activity of BCRP during Ko143 treatment was still sufficient to limit the transfer of glyburide from the maternal to the fetal compartments.

\section{Conclusion}

In this work, we have demonstrated the feasibility of utilizing the placenta-on-a-chip platform for analysis of drug transport across the human placental barrier. Using glyburide as a representative medication, our data show the capability of the placenta-on-a-chip to recapitulate the native function of efflux transporters and to mimic the limited placental transfer of a maternally administered drug. While the physiological relevance of this model has yet to be validated in a more systematic and rigorous manner, our findings are consistent with previous reports of glyburide transport in the human placenta. For example, the first randomized human trial to compare glyburide and insulin in women with gestational diabetes showed that glyburide levels were undetectable in the cord serum of any infant ${ }^{[30]}$. Similarly, studies using the gold standard ex vivo perfusion of the human placenta reported uniformly low glyburide transport rates ranging from $0.62 \%$ to $3.9 \%$, which approximates those estimated in our model $(5.6 \%)^{[28,29]}$. 
Despite the demonstrated capabilities of our model, further studies are needed to maximize its potential for the study of placental drug transport. Of particular importance is that our system has been designed to mimic the placental barrier late in pregnancy, when there is minimal distance between syncytiotrophoblasts and fetal capillaries ${ }^{[13]}$. Thus, this model is not appropriate for modeling drug transport in earlier stages of gestation. Given the plasticity of placental structure, new engineering strategies need to be developed to reconstitute the significant changes that occur in the microarchitecture of the human placental barrier throughout pregnancy. For example, it may be possible to create a model that incorporates cytotrophoblasts and interstitial stromal tissue to study the little known process of transplacental drug transfer in the first trimester. Another important goal for future investigation is to extend our analysis to other key transporters reported in the human placenta and to evaluate the transfer of their substrate drugs in our model. These types of studies may be limited by the transformed cells (BeWo) used in our current system that fail to fully recapitulate the expression profiles of native transporters. To address this problem, work is required to incorporate stable culture of primary human villous trophoblast cells into our system. Primary villous trophoblasts can be isolated from term placentas using protocols similar to those developed for isolation of HPVECs. Since these cells are post-mitotic and cannot be externally stimulated to proliferate, however, it is challenging to establish and maintain confluent trophoblast monolayers. Furthermore, the behavior of primary trophoblast cells under perfusion culture conditions has not been investigated previously. Future studies are needed to address these potentially significant challenges and to develop new strategies for generation and long-term maintenance of structurally stable primary trophoblast barrier in microfluidic cell culture devices. Finally, special attention needs to be paid to the absorption of drug compounds into PDMS. This undesirable property is a particularly critical issue for drug screening that can significantly alter the pharmacokinetics of small molecule therapeutics. Studies are necessary to explore alternative materials or develop new strategies (e.g., engineering of PDMS surface chemistry) to render cell culture chambers resistant to drug absorption.

In conclusion, our work represents a significant advance that may provide the impetus to improve and innovate traditional models in reproductive toxicology. With increasing use of medications during pregnancy, the paucity of preclinical models for predicting drug transfer in the human placenta is emerging as a critical research challenge that also raises serious public health concerns. The proof-of-principle demonstrated in this paper may hold great potential as a technical basis for developing new drug screening technologies to address this important problem. Specifically, our model, with its current capabilities, may find obvious and immediate applications in screening the likelihood of fetal exposure to a wide variety of drug compounds currently in use or under development for use in pregnancy. Beyond studies of placental drug transport, this platform also provides a robust means to systematically investigate how the structure and function of the placental barrier itself can be altered in response to perturbations in the local environment due to various factors including drugs, xenobiotics, pathogens, abnormal hemodynamics, disease-derived biomolecules, and environmental toxicants. Another possibility to explore building upon recent progress in the development of multi-organ microphysiological models is to link the placenta-on-a-chip with other microfluidic devices containing different types of fetal cells for analysis of not 
only placental transfer of drug compounds but also their beneficial or adverse health effects on the developing fetus. We believe that the versatility of our placenta-on-a-chip technology offers great promise for enabling significant progress in various aspects of placental physiology and reproductive medicine.

\section{Experimental Section}

\section{Cell Culture}

The $\mathrm{b} 30$ clone of the BeWo trophoblast cell line was generously provided by Dr. Nicholas Illsley of Hackensack University Medical Center. The BeWo cells were maintained in DMEM/F-12K medium (GE Healthcare) containing 10\% fetal bovine serum (FBS), $1 \%$ Lglutamine and $1 \%$ penicillin/streptomycin (Gibco). Human placental villous endothelial cells (HPVECs) isolated from term placentas following a previously published protocol ${ }^{[35]}$ were generously provided by Dr. Emily Su at the University of Colorado, Denver and cultured in EGM-2 media containing 2\% FBS (Lonza).

\section{Microfluidic Device Fabrication}

Placenta-on-a-chip devices were fabricated following standard soft lithography protocols. Poly(dimethylsiloxane) (PDMS) (Sylgard, Dow Corning) base was mixed thoroughly with curing agent at a ratio of 10:1. This mixture was then poured over a photolithographically prepared silicon master containing microchannel features fabricated in a negative photoresist, SU-8 (MicroChem). The dimensions of the microchannels were $1 \mathrm{~mm}$ (width) $\times$ $1.5 \mathrm{~cm}$ (length) $\times 135 \mu \mathrm{m}$ (height). The PDMS was degassed and then incubated at $65 \mathrm{C}$ to induce polymerization. Once fully cured, the channel slab was peeled off of the master, and through-holes were made through the slab using a $1 \mathrm{~mm}$ biopsy punch to generate inlet and outlet access ports.

For device assembly, PDMS stamping was used to bond the upper and lower slabs to a semipermeable polycarbonate membrane ( $1 \mu \mathrm{m}$ pores, GE Healthcare) as previously described $^{[36]}$. In this technique, the upper channel slab was stamped onto a thin $(\sim 10 \mu \mathrm{m})$ layer of uncured PDMS and then bonded to the lower channel slab. During this step, the intervening membrane was sandwiched between the two channel layers.

\section{Microfluidic Cell Culture}

Following fabrication, the PDMS microchannels were filled with $70 \%$ ethanol and incubated for 1 minute. The ethanol solution was then removed from the channels by vacuum aspiration applied to the access ports of the upper and lower microchannels. This step was repeated until complete removal of the solution was achieved. The device was then placed under ultraviolet light for a 20-minute sterilization cycle. Next, to prepare the polycarbonate membrane for cell seeding, the microchannels were filled with human fibronectin solution $(0.1 \mathrm{mg} / \mathrm{ml}$ in PBS) and incubated at $37 \mathrm{C}$ for a minimum of 4 hours. This solution was rinsed with PBS prior to the introduction of cells. Trypsinized HPVECs at a concentration of $4 \times 10^{6} \mathrm{cells} / \mathrm{ml}$ were first introduced into the lower microchannel. The device was inverted and incubated for 1 hour in a cell culture incubator to enable attachment of the cells to the lower surface of the membrane. Following endothelial cell adhesion, BeWo cells were 
subcultured and injected into the upper microchannel at a concentration of $4 \times 10^{6}$ cells $/ \mathrm{ml}$. The microdevice was again incubated at $37 \mathrm{C}$ for at least 1 hour for BeWo cell attachment. Once complete cell attachment was confirmed, the device was connected to a syringe pump (Chemyx) for continuous perfusion at a flow rate of $100 \mu \mathrm{l} / \mathrm{h}$. To induce syncytialization, the BeWo cells in the upper microchannel was perfused with DMEM/F12-K medium (GE Healthcare) containing $50 \mu \mathrm{M}$ forskolin (Sigma). The lower microchannel containing HPVECs was perfused with EGM-2 medium (Lonza).

\section{Barrier Characterization}

Cells cultured in our microfluidic devices were fixed in 4\% paraformaldehyde (PFA) for 15 minutes and permeabilized in $0.25 \%$ Triton X-100 for 10 minutes, with thorough rinse steps in between. Next, the channels were filled with $2 \%$ bovine serum albumin (BSA) for 1 hour at room temperature. For imaging of intercellular junctions, BeWo cells were incubated with anti-E-cadherin (Life Technologies) and HPVECs were incubated with anti-VE-cadherin (Cell Signaling Technologies) for 1 hour. Following a PBS rinse, secondary antibodies diluted in 2\% BSA (Life Technologies) were introduced into the microdevice and incubated for 45 minutes. For visualization of trophoblast microvilli and the actin cytoskeleton, cells were exposed to Alexa Fluor 488 conjugated phalloidin (Life Technologies) for 30 minutes. Finally, DAPI was utilized to stain cell nuclei. Immunofluorescent images were acquired using a confocal laser-scanning microscope (Leica TCS SP8) and inverted microscope (Zeiss Axio Observer). Volocity (PerkinElmer) was utilized for image processing.

\section{Analysis of Cell Fusion}

Fusion of BeWo cells following forskolin treatment was analyzed using MATLAB and CellProfiler. These values were calculated from 3-5 images at each time point. A MATLAB script was utilized to segment nuclei based on thresholding and subsequently quantify nuclear characteristics, including area, in pixels. Changes in nuclear area indicated aggregation and fusion of BeWo cell nuclei. Junction segmentation was performed with CellProfiler by applying maximum correlation thresholding to the E-cadherin channel followed by intensity-based object identification and de-clumping ${ }^{[37,38]}$. By segmenting based on E-cadherin expression, the area of individual cells could be measured. The quantification of changes in cell area enabled analysis of progressive forskolin-induced cell fusion in the BeWo cell population.

\section{TEER measurements}

A digital voltage-ohm multimeter (Fluke Corporation) was used to measure TEER under coculture conditions. The electrodes were placed into the outlet tubing of each channel for analysis. Baseline measurements were made in acellular devices and subtracted from the results in the co-culture devices.

\section{Permeability assay}

Fluorescein isothiocyanate-inulin (FITC-inulin, Sigma) was diluted to a concentration of 1 $\mathrm{mg} / \mathrm{mL}$ in serum-free DMEM/F12K medium. This solution was perfused through the upper 
microchannel, and perfusates from both microchannels were assessed following 3 hours using a microplate reader (Tecan).

\section{Measurement of Fluorescein-Heparin Transport}

Green-fluorescent fluorescein heparin conjugate (Thermo Fisher) was diluted to a concentration of $0.05 \mathrm{mg} / \mathrm{mL}$ in DMEM/F12K medium. The fluorescent heparin solution was perfused through the upper microchannel, and outflow was collected from both channels following a 5-hour perfusion. The duration of drug perfusion was chosen to ensure sufficient sample volumes for transport analysis. Fluorescence levels of the maternal and fetal channel perfusates were quantified using a microplate reader (Tecan). These steps were carried out in both co-culture and bare membrane (acellular) conditions on-chip.

\section{Analysis of Glyburide Transport}

For both monoculture and co-culture glyburide transport studies, BODIPY-conjugated glyburide was diluted into serum-free DMEM/F-12K medium and used at a final concentration of $1 \mu \mathrm{M}$. The microdevice was connected to a syringe pump for infusion, with drug-containing medium perfused through the upper microchannel and serum-free EGM-2 medium in the lower microchannel. Following a 30-minute equilibration phase, outflow was serially collected in 30 minute intervals, and fluorescence was quantified using a microplate reader (Tecan). Where appropriate, a standard curve was generated to enable calculation of drug concentrations from mean fluorescence intensity of each sample. Following the time course study, the microdevice was imaged using an inverted microscope (Zeiss Axio Observer) to assess intracellular drug accumulation. For inhibitor studies, Ko143 (150 nM in DMSO, Sigma) was added to the drug-containing medium perfused continuously in the upper microchannel. Each transport study was repeated in 6 devices.

\section{Statistical analysis}

Results were presented as mean \pm S.E.M. Statistical analysis was carried out using a twotailed Student's t-test.

\section{Supplementary Material}

Refer to Web version on PubMed Central for supplementary material.

\section{Acknowledgments}

This work was supported by the March of Dimes Prematurity Research Center at the University of Pennsylvania, the NIH Director's New Innovator Award (D.H.; 1DP2HL127720-01), NIH R01ES020522 (L.M.A), and NIH P30ES005022 (L.M.A). We thank N.P. Illsley, A. Schwartz, J. Seo, M. Mondrinos for their input to this study.

\section{References}

1. Mitchell AA, Gilboa SM, Werler MM, Kelley KE, Louik C, Hernández-Díaz S. National Birth Defects Prevention Study. American Journal of Obstetrics and Gynecology. 2011; 205:51.e1. [PubMed: 21514558]

2. Werler MM, Mitchell AA, Hernández-Díaz S, Honein MA. the National Birth Defects Prevention Study. American Journal of Obstetrics and Gynecology. 2005; 193:771. [PubMed: 16150273] 
3. Gude NM, Roberts CT, Kalionis B, King RG. Thrombosis Research. 2004; 114:397. [PubMed: 15507270]

4. Grigsby PL. Semin. Reprod. Med. 2016; 34:11. [PubMed: 26752715]

5. Sheffield JS, Siegel D, Mirochnick M, Heine RP, Nguyen C, Bergman KL, Savic RM, Long J, Dooley KE, Nesin M. Clinical Infectious Diseases. 2014; 59:S437. [PubMed: 25425722]

6. Miller MT, Strömland K. Teratology. 1999; 60:306. [PubMed: 10525208]

7. Blundell C, Tess ER, Schanzer ASR, Coutifaris C, Su EJ, Parry S, Huh D. Lab Chip. 2016; 16:3065. [PubMed: 27229450]

8. Poulsen MS, Rytting E, Mose T, Knudsen LE. Toxicology in Vitro. 2009; 23:1380. [PubMed: 19647068]

9. Brandes JM, Tavoloni N, Potter BJ, Sarkozi L, Shepard MD, Berk PD. The American Journal of Obstetrics \& Gynecology. 1983; 146:800. [PubMed: 6869452]

10. Mathiesen L, Mose T, Mørck TJ, Nielsen JKS, Nielsen LK, Maroun LL, Dziegiel MH, Larsen LG, Knudsen LE. Reproductive Toxicology. 2010; 30:138. [PubMed: 20096346]

11. Maliepaard M, Scheffer GL, Faneyte IF, van Gastelen MA, Pijnenborg AC, Schinkel AH, van de Vijver MJ, Scheper RJ, Schellens JH. Cancer Research. 2001; 61:3458. [PubMed: 11309308]

12. Mao Q. Pharm. Res. 2008; 25:1244. [PubMed: 18202831]

13. Feneley MR, Burton GJ. Placenta. 1991; 12:131. [PubMed: 1871071]

14. Enders A, Blankenship T. Adv. Drug Deliv. Rev. 1999; 38:3. [PubMed: 10837743]

15. Lager S, Powell TL. J Pregnancy. 2012; 2012:179827. [PubMed: 23304511]

16. Teasdale F. The American Journal of Obstetrics \& Gynecology. 1980; 137:560. [PubMed: 7386550]

17. Syme MR, Paxton JW, Keelan JA. Clin Pharmacokinet. 2004; 43:487. [PubMed: 15170365]

18. Keryer G, Alsat E, Tasken K, Evain-Brion D. Journal of Cell Science. 1998; 111(Pt 7):995. [PubMed: 9490643]

19. Wice B, Menton D, Geuze H, Schwartz AL. Exp. Cell Res. 1990; 186:306. [PubMed: 2153559]

20. Odijk M, van der Meer AD, Levner D, Kim HJ, van der Helm MW, Segerink LI, Frimat J-P, Hamilton GA, Ingber DE, van den Berg A. Lab Chip. 2015; 15:745. [PubMed: 25427650]

21. Oakley CM. Br Heart J. 1995; 74:107. [PubMed: 7546985]

22. Deruelle P, Coulon C. Curr. Opin. Obstet. Gynecol. 2007; 19:573. [PubMed: 18007136]

23. Mätzsch T, Bergqvist D, Bergqvist A, Hodson S, Dawes J, Hedner U, Ostergaard P. Blood Coagul. Fibrinolysis. 1991; 2:273. [PubMed: 1893059]

24. Forestier F, Daffos F, Rainaut M, Toulemonde F. Thromb. Haemost. 1987; 57:234. [PubMed: 3603416]

25. Dimitrakakis C, Papageorgiou P, Papageorgiou I, Antzaklis A, Sakarelou N, Michalas S. Haemostasis. 2000; 30:243. [PubMed: 11251331]

26. DeSisto CL, Kim SY, Sharma AJ. Prev. Chronic Dis. 2014; 11:130415.

27. Ferrara A. Diabetes Care. 2007; 30:S141. [PubMed: 17596462]

28. Elliott BD, Langer O, Schenker S, Johnson RF. The American Journal of Obstetrics \& Gynecology. 1991; 165:807. [PubMed: 1951536]

29. Elliott BD, Schenker S, Langer O, Johnson R, Prihoda T. The American Journal of Obstetrics \& Gynecology. 1994; 171:653. [PubMed: 8092211]

30. Langer O, Conway DL, Berkus MD, Xenakis EM, Gonzales O. N Engl J Med. 2000; 343:1134. [PubMed: 11036118]

31. Iqbal M, Audette MC, Petropoulos S, Gibb W, Matthews SG. Placenta. 2012; 33:137. [PubMed: 22265855]

32. Toepke MW, Beebe DJ. Lab Chip. 2006; 6:1484. [PubMed: 17203151]

33. Matsson PR, Pedersen JM, Norinder U, Bergstr m CAS, Artursson P. Pharm. Res. 2009; 26:1816. [PubMed: 19421845]

34. Bircsak KM, Gibson CJ, Robey RW, Aleksunes LM. Curr Protoc Toxicol. 2013; 57(Unit 23.6)

35. Su EJ, Cheng Y-H, Chatterton RT, Lin Z-H, Yin P, Reierstad S, Innes J, Bulun SE. Biology of Reproduction. 2007; 77:517. [PubMed: 17538076] 
36. Chueh B-H, Huh D, Kyrtsos CR, Houssin T, Futai N, Takayama S. Analytical chemistry. 2007; 79:3504. [PubMed: 17388566]

37. Kamentsky L, Jones TR, Fraser A, Bray M-A, Logan DJ, Madden KL, Ljosa V, Rueden C, Eliceiri KW, Carpenter AE. Bioinformatics. 2011; 27:1179. [PubMed: 21349861]

38. Carpenter AE, Jones TR, Lamprecht MR, Clarke C, Kang IH, Friman O, Guertin DA, Chang JH, Lindquist RA, Moffat J, Golland P, Sabatini DM. Genome Biol. 2006; 7:R100. [PubMed: 17076895] 


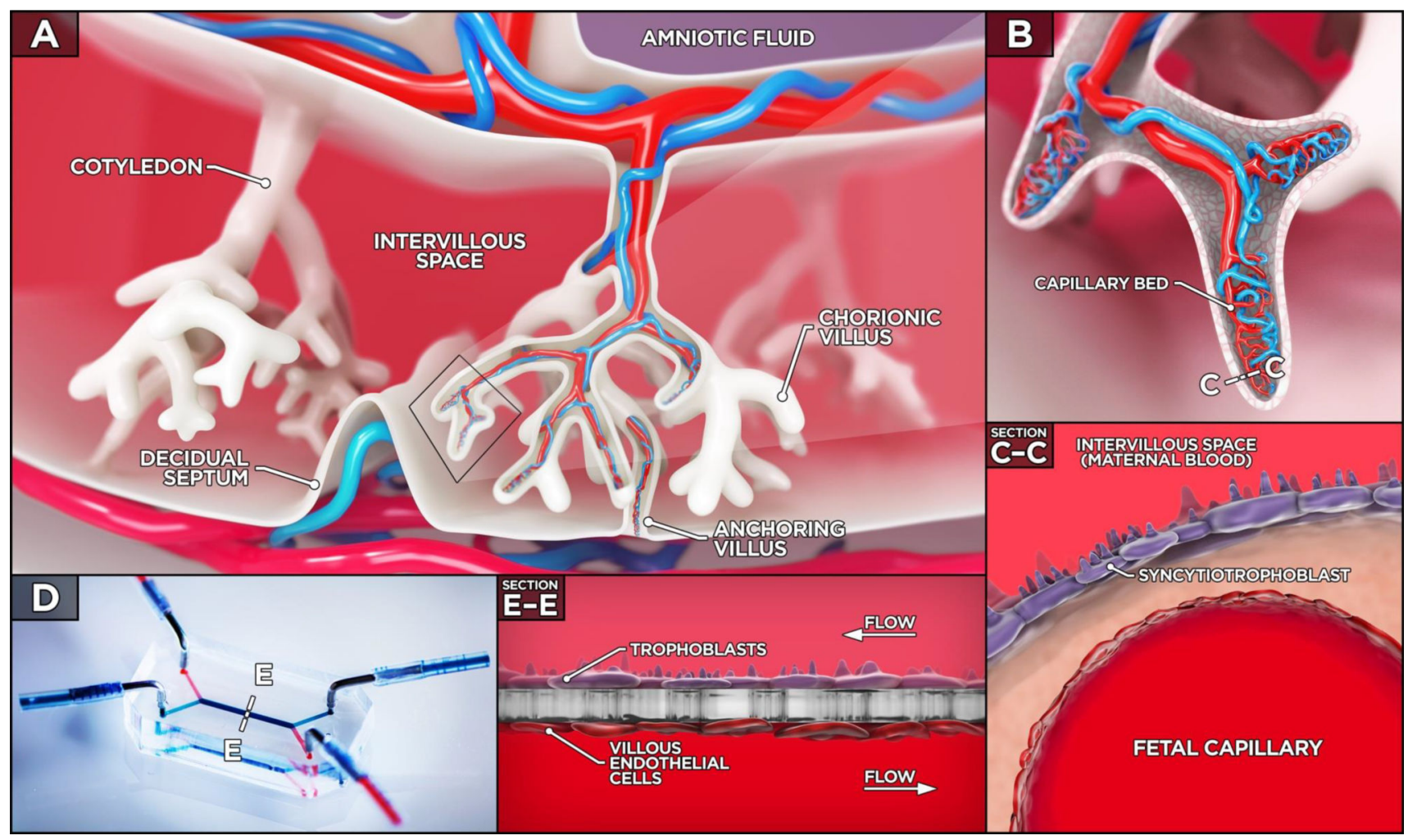

Figure 1. The human placenta and placenta-on-a-chip

A. Three-dimensional cross section of the human placenta illustrates cotyledons, which contain chorionic villi bathed in the maternal blood in the intervillous space. B. A zoomedin view highlights the fetal capillaries that are located within the chorionic villi. $\mathbf{C}$. The placental barrier that separates the maternal intervillous space from the fetal capillary lumen consists of two cell types: the syncytiotrophoblast and fetal endothelial cells separated by a thin interstitial tissue layer. D-E. The placenta-on-a-chip is a microengineered model designed to recapitulate the multi-layered three-dimensional architecture of the placental barrier. Within the device, trophoblast and endothelial cells are co-cultured on the opposite sides of a thin porous polymeric membrane. During culture, the viability of the cells is maintained by continuous low of culture medium on both sides of the membrane. 

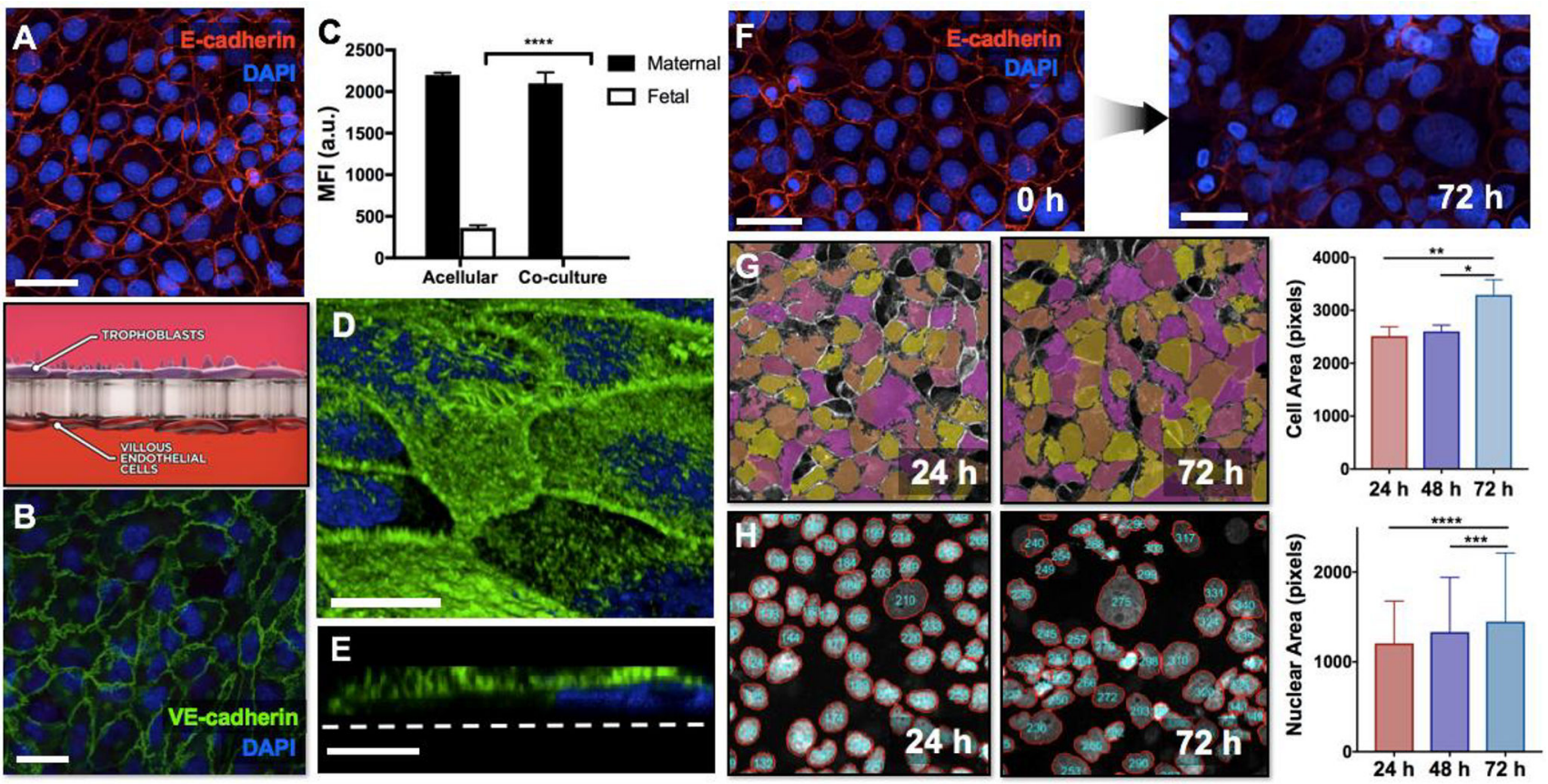

Figure 2. Microengineered in vitro placental barrier

A. BeWo cells cultured on the upper side of the porous membrane form a continuous epithelial barrier. Red shows E-cadherin staining. Scale bar: $55 \mu \mathrm{m}$. B. Human placental villous endothelial cells (HPVECs) are grown to confluence on the lower side of the membrane. Widespread expression of VE-cadherin (green) illustrates the structural integrity of the endothelial tissue. Scale bar: $36 \mu \mathrm{m}$. C. The microengineered placental barrier effectively prevents the transfer of FITC-inulin from the maternal microchannel to the fetal compartment, demonstrating appropriate barrier function. D. Dense microvilli cover the surface of the BeWo cell population, as illustrated by F-actin staining (green). Scale bar: 20 $\mu \mathrm{m}$. E. A cross-sectional view of actin-stained BeWo cells reveals the apical microvilli projections protruding from the cell body. Scale bar: $6 \mu \mathrm{m}$. F. Extended forskolin treatment induces a significant loss of intercellular junctions (red) and syncytialization-like cell fusion in BeWo cells over the course of 72 hours. Scale bars: $55 \mu \mathrm{m}$. Blue shows nuclear staining. G. During image analysis of E-cadherin staining, individual cells are pseudo-colored to delineate cell-cell junctions and to quantify cell area during forskolin treatment. The average area of BeWo cells increases over 72 hours, illustrating progressive cell fusion in the trophoblast population. * and $* *$ indicate $\mathrm{p}<0.05$ and $\mathrm{p}<0.01$, respectively. H. Another method for image analysis of cell fusion is to segment DAPI-stained nuclei of BeWo cells and then measure the area of the segmented nuclei. The average size of cell nuclei increases during forskolin treatment. $* * *$ and $* * * *$ indicate $\mathrm{p}<0.001$ and $\mathrm{p}<0.0001$, respectively. 

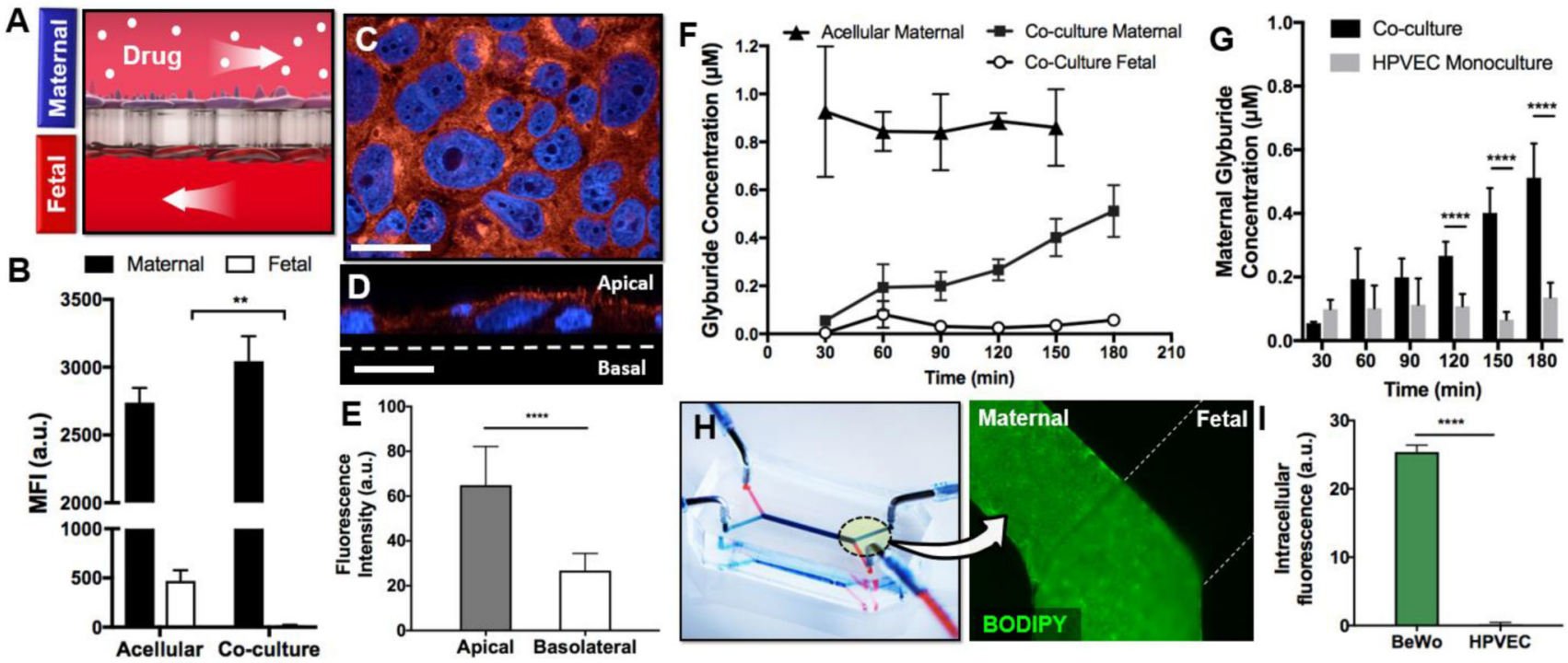

Figure 3. Drug transport in the placenta-on-a-chip

A. The kinetics of drug transport across the microengineered placental barrier is examined by perfusing the maternal microchannel with fluorescently labeled substrate and measuring fluorescence in the perfusates. B. Transfer of fluorescein-heparin from the maternal compartment to the fetal chamber is evaluated by measuring mean fluorescence intensity (MFI) of perfusate collected from the fetal microchannel. Acellular devices allow a significant fraction of maternally administered heparin to move across the membrane into the fetal compartment, whereas the barrier covered with trophoblast cells and endothelial cells (co-culture) effectively blocks heparin transport. ** shows $\mathrm{p}<0.01$. C. BeWo cells exhibit widespread expression of BCRP (red). Blue shows cell nuclei. Scale bar: $36 \mu \mathrm{m}$. D. BCRP is localized to the maternal-facing microvillous membrane of BeWo cells. **** shows $\mathrm{p}<0.0001$. Scale bar: $36 \mu \mathrm{m}$. F. In the co-culture model, a continuous increase in the fluorescence of maternal perfusate indicates transporter-mediated efflux of glyburide back into the maternal circulation. The fluorescence of perfusate from the fetal compartment remains low and constant, showing limited transfer. G. Under endothelial cell monoculture conditions, maternal glyburide concentration is decreased. $* * * *$ indicates $\mathrm{p}<0.0001$. H. Microscopic analysis illustrates accumulation of glyburide in the BeWo cell monolayer, but no visible fluorescence in the HPVEC population. $* * * *$ indicates $\mathrm{p}<0.0001$. 

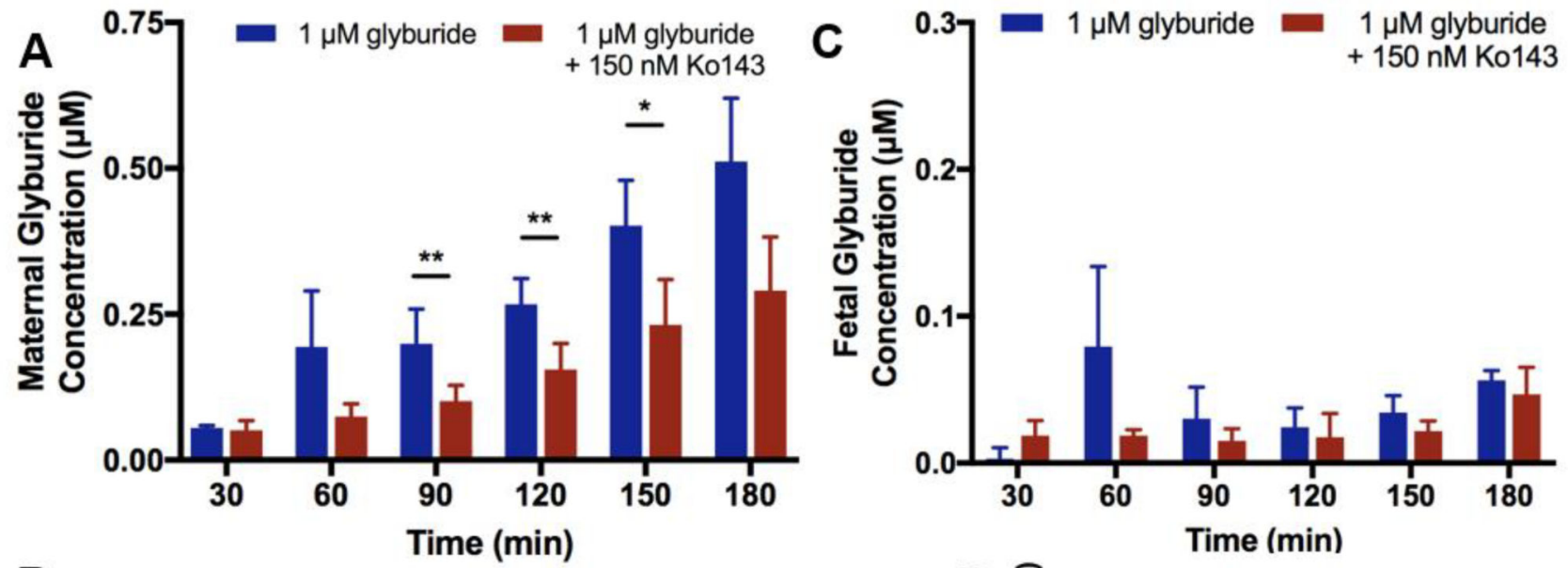

\section{No Ko143}

BODIPY

\section{$150 \mathrm{nM}$ Ko143}

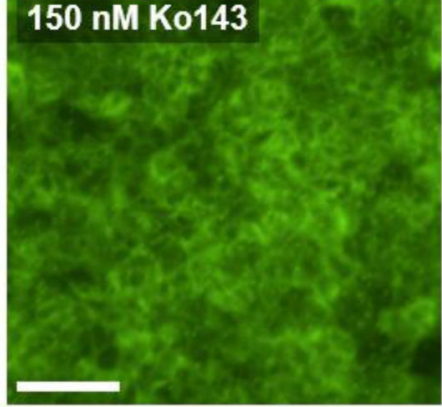

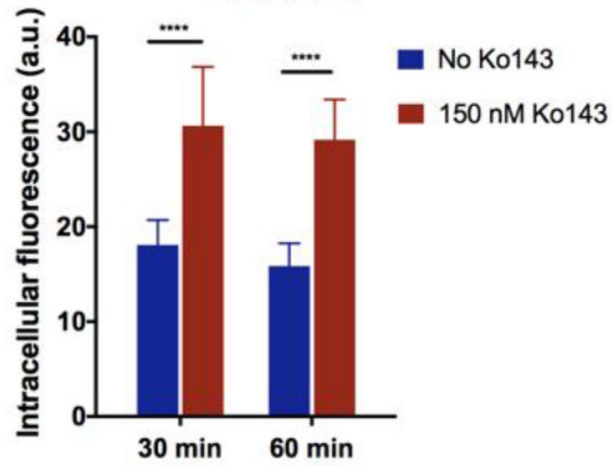

Figure 4. Inhibition of BCRP

A. Addition of Ko143, a BCRP inhibitor, results in decreased maternal glyburide levels as compared to the control perfusion. This is attributable to inhibition of efflux function of BCRP. B. The levels of fetal glyburide in the inhibitor condition are not significantly different from those in the control group without the inhibitor. Passage of glyburide to the fetal compartment remains very low over the course of drug perfusion in both groups. C. Increased drug accumulation in BeWo cells due to reduced activity of BCRP is evident from fluorescence imaging of the cells. D. In the presence of the inhibitor, the cells show significantly higher levels of fluorescence, suggesting increased intracellular drug retention. $* * * *$ indicates $\mathrm{p}<0.0001$. 